\title{
Flap Capillary Blood Glucose Monitoring As a Predictor of Flap Survival in Below Knee Amputation Stump
}

\author{
S.Muthulakshmi ${ }^{1}$, M.Muralidaran ${ }^{2}$, \\ ${ }^{1}$ Asst Professor, Department of General Surgery, Coimbatore, TN, India \\ ${ }^{2} \mathrm{Pg}$, Department Of General Surgery, Coimbatore, TN, India
}

\begin{abstract}
Monitoring of flap blood glucose levels during and after surgery is important for determining flap salvage or loss. Decision to re operate and do revision amputation was solely based on clinical assessment and no objective measurement is available till date. The objective of this study is to validate flap capillary blood glucose monitoring serially as an indicator to predict flap survival in below knee amputation patients. The study was conducted at Coimbatore medical college hospital, general surgery department from march-2016 to june2016. 50 patients with peripheral arterial occlusive disease with established gangrene of lower limb requiring $B K$ amputations were selected. Out of the 50 patients who underwent $B K$ amputations 45 patients in whom the flap survived had flap glucose level more than $62 \mathrm{mg} / \mathrm{dl}$, in the first 24 hours. This simple and cheap technique can be used for routine monitoring of BK amputation flaps along with the routine clinical evaluation.
\end{abstract}

Keywords: BKflaps, glucose, gangrene, flap survival

\section{Introduction}

Flap failure in BK amputation ${ }^{1}$ leads to serious morbidities. The current monitoring process for assessing flap perfusion remains mostly subjective. The objective of this study is to measure capillary glucose levels using glucometer in detecting post operative flap tissue ischemia in patients undergoing BK amputations. This is an efficient, simple and cheap technique to detect early post operative flap ischemia in BK flaps.

\section{Methods}

The study was conducted at Coimbatore medical college hospital at department of general surgery for a period of 4 months from march-16 to june-16 after the institutional ethical committee approval.

50 patients with peripheral arterial disease ${ }^{2}$ with established gangrene of lower limb requiring BK amputations were selected. Patients below 18 years, pregnant patients and traumatic amputations ${ }^{3}$ were excluded.

Doppler ultrasonography of the posterior tibial vascular pedicle ${ }^{4}$ based flaps with good tissue oxygenation $^{5}$ was selected. A drop of blood was taken from the flap and from the finger tip using a needle and blood glucose measurements were done serially at 0,6,24 hours using the same glucometer device. The data were analysed . the flaps were clinically also assessed as per protocol ${ }^{6}$. The finger capillary blood glucose level will be used as a control for the flap blood glucose level.

\section{Results}

Of the 50 patients who underwent BK amputations, 5 patients developed postoperative flap necrosis due to venous thrombosis ${ }^{7}$ and developed gangrene leading to revision amputation. 6 patients developed flap infection which was treated with appropriate antibiotics after culture and sensitivity and the flap survived. Rest of the 39 patients had healthy flaps and uneventful postoperative period.

Correlation between the clinical observation and glucose level measurement can be seen from the below table. Figure 1 illustrates flap glucose levels at 0,6,24 hours in patients who developed gangrene. 
Flap Capillary Blood Glucose Monitoring As A Predictor Of Flap Survival In Below Knee..

Figure-1.

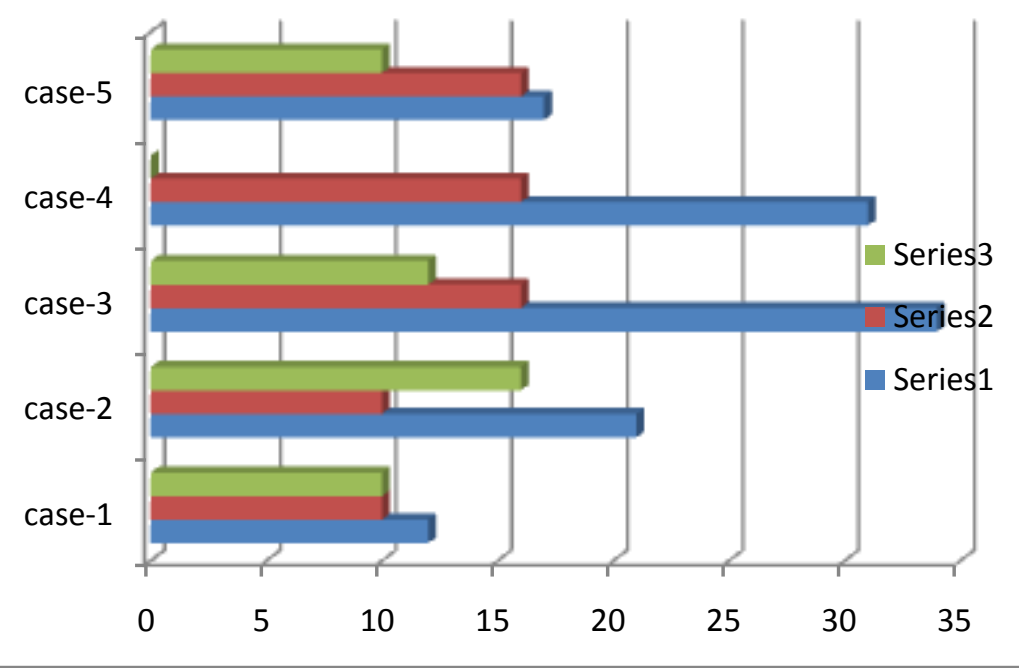

\begin{tabular}{|c|c|c|c|c|c|c|c|}
\hline \multicolumn{8}{|c|}{ Table-1 } \\
\hline \multirow{3}{*}{ s.no } & \multirow{2}{*}{\multicolumn{3}{|c|}{ flap-cbg }} & \multirow{2}{*}{\multicolumn{3}{|c|}{ finger-cbg }} & \multirow{3}{*}{ outcome } \\
\hline & & & & & & & \\
\hline & 0 & 6 & 24 & 0 & 6 & 24 & \\
\hline 1 & 87 & 82 & 92 & 92 & 90 & 103 & survived \\
\hline 2 & 69 & 66 & 74 & 72 & 76 & 68 & survived \\
\hline 3 & 77 & 72 & 76 & 82 & 77 & 76 & survived \\
\hline 4 & 102 & 98 & 122 & 122 & 124 & 124 & survived \\
\hline 5 & 111 & 121 & 112 & 126 & 128 & 124 & survived \\
\hline 6 & 56 & 87 & 88 & 98 & 92 & 92 & survived \\
\hline 7 & 78 & 68 & 76 & 82 & 86 & 78 & survived \\
\hline 8 & 98 & 86 & 99 & 102 & 111 & 104 & survived \\
\hline 9 & 90 & 100 & 112 & 122 & 106 & 112 & survived \\
\hline 10 & 45 & 68 & 72 & 72 & 72 & 68 & survived \\
\hline 11 & 12 & 10 & 10 & 68 & 72 & 68 & gangrene \\
\hline 12 & 87 & 122 & 92 & 98 & 122 & 102 & infected,survived \\
\hline 13 & 96 & 78 & 88 & 121 & 102 & 98 & survived \\
\hline 14 & 112 & 102 & 98 & 121 & 98 & 106 & survived \\
\hline 15 & 99 & 78 & 88 & 102 & 88 & 89 & survived \\
\hline 16 & 85 & 78 & 98 & 121 & 118 & 104 & infected,survived \\
\hline 17 & 21 & 10 & 16 & 102 & 98 & 121 & gangrene \\
\hline 18 & 79 & 68 & 87 & 102 & 98 & 88 & survived \\
\hline 19 & 121 & 126 & 130 & 132 & 128 & 132 & survived \\
\hline 20 & 89 & 86 & 92 & 104 & 112 & 106 & survived \\
\hline 21 & 94 & 98 & 104 & 108 & 126 & 112 & survived \\
\hline 22 & 78 & 82 & 69 & 86 & 92 & 89 & survived \\
\hline 23 & 94 & 78 & 83 & 96 & 88 & 98 & survived \\
\hline 24 & 124 & 112 & 102 & 134 & 126 & 126 & survived \\
\hline 25 & 99 & 78 & 86 & 112 & 104 & 100 & survived \\
\hline 26 & 34 & 16 & 12 & 68 & 78 & 76 & gangrene \\
\hline 27 & 122 & 88 & 121 & 136 & 121 & 102 & infected,survived \\
\hline 28 & 88 & 78 & 86 & 132 & 98 & 96 & survived \\
\hline 29 & 93 & 82 & 86 & 96 & 98 & 92 & survived \\
\hline 30 & 132 & 102 & 121 & 136 & 126 & 138 & survived \\
\hline 31 & 63 & 72 & 68 & 68 & 76 & 76 & survived \\
\hline 32 & 82 & 86 & 76 & 98 & 96 & 98 & infected,survived \\
\hline 33 & 72 & 68 & 76 & 89 & 102 & 78 & survived \\
\hline 34 & 59 & 79 & 86 & 96 & 68 & 98 & survived \\
\hline
\end{tabular}


Flap Capillary Blood Glucose Monitoring As A Predictor Of Flap Survival In Below Knee..

\begin{tabular}{|l|l|l|l|l|l|l|l|}
\hline 35 & 67 & 72 & 74 & 72 & 82 & 78 & infected,survived \\
\hline 36 & 58 & 64 & 66 & 72 & 72 & 68 & survived \\
\hline 37 & 92 & 84 & 78 & 102 & 98 & 88 & survived \\
\hline 38 & 71 & 78 & 82 & 89 & 92 & 90 & survived \\
\hline 39 & 73 & 67 & 72 & 82 & 84 & 82 & survived \\
\hline 40 & 64 & 72 & 72 & 82 & 76 & 78 & survived \\
\hline 41 & 77 & 66 & 72 & 82 & 86 & 86 & survived \\
\hline 42 & 81 & 72 & 98 & 124 & 112 & 120 & infected,survived \\
\hline 43 & 59 & 62 & 64 & 78 & 82 & 66 & survived \\
\hline 44 & 31 & 16 & $0($ low $)$ & 88 & 96 & 98 & gangrene \\
\hline 45 & 56 & 62 & 58 & 68 & 66 & 72 & survived \\
\hline 46 & 17 & 16 & 10 & 68 & 86 & 66 & gangrene \\
\hline 47 & 98 & 86 & 102 & 121 & 112 & 124 & survived \\
\hline 48 & 63 & 66 & 72 & 86 & 78 & 88 & survived \\
\hline 49 & 72 & 74 & 66 & 82 & 77 & 76 & survived \\
\hline 50 & 66 & 64 & 72 & 72 & 66 & 66 & survived \\
\hline
\end{tabular}

Figure 2 illustrates mean flap capillary glucose levels at 0, 6, and 24 hours.

Figure 2 (a) At 0 hour:

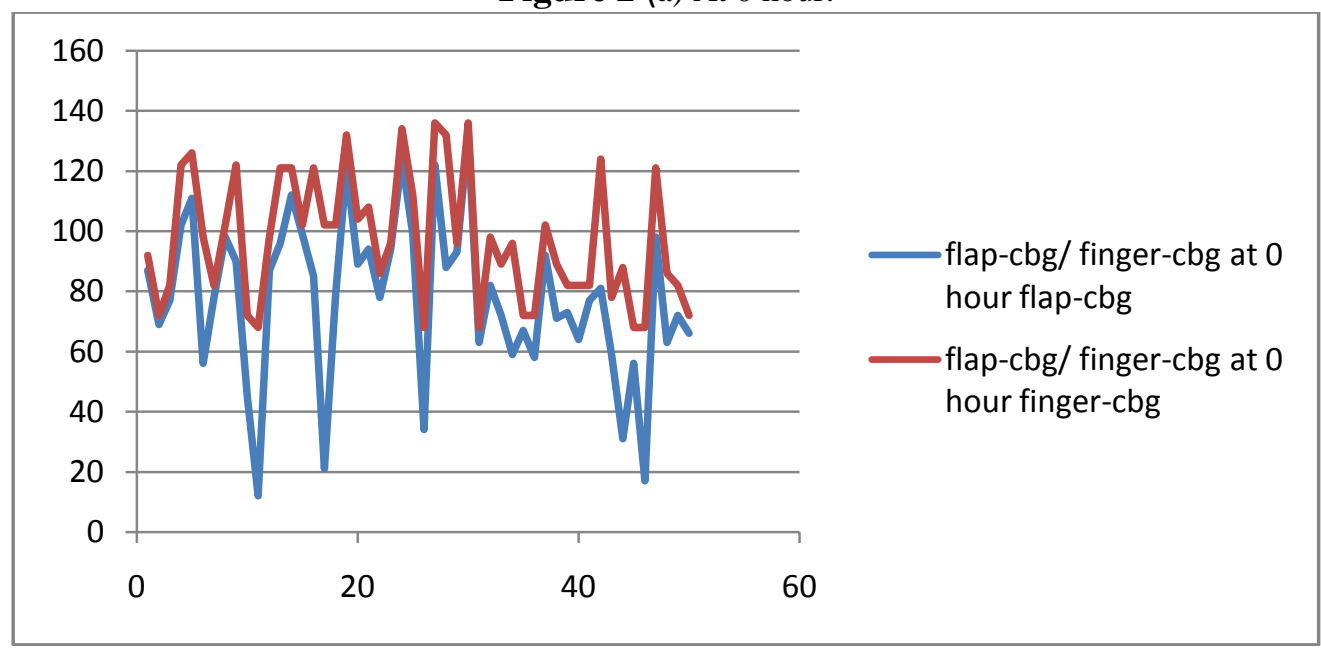

(b) At 6 hours:

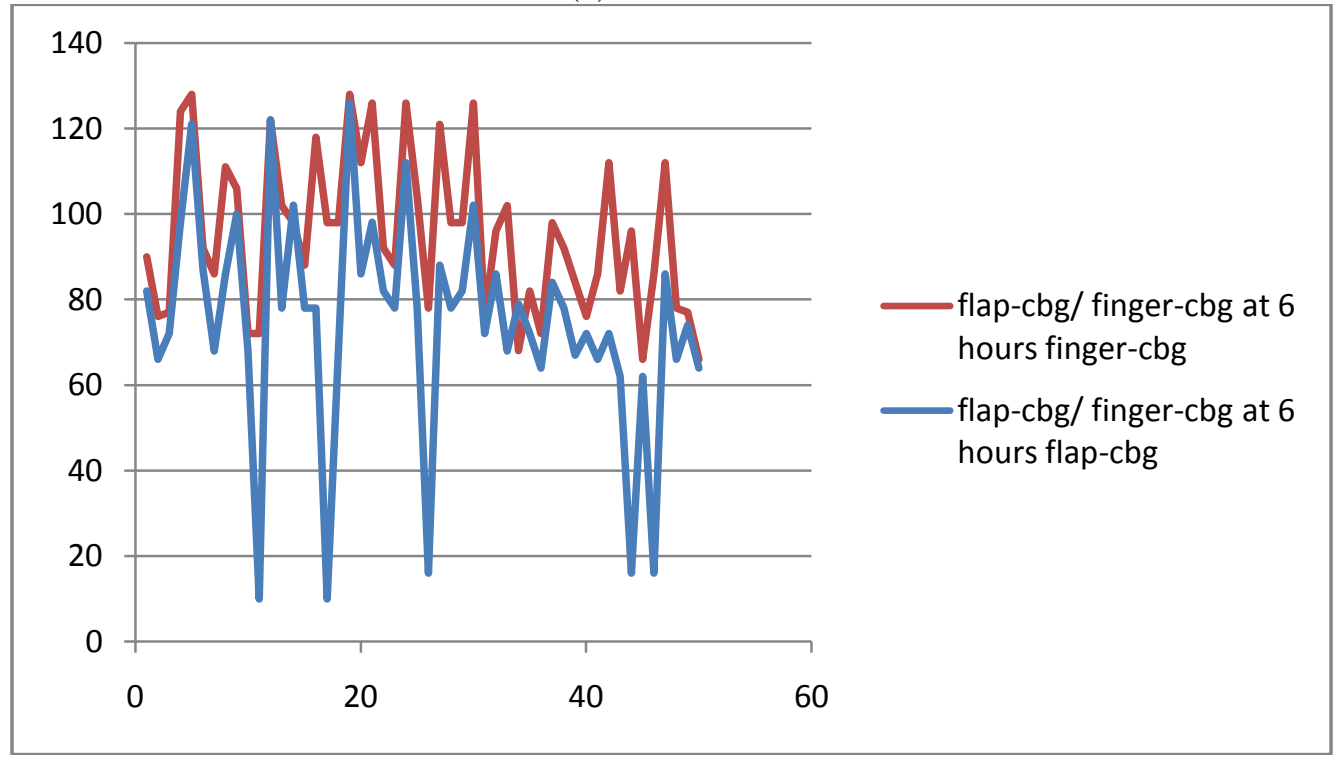

(c) At 24 hours: 


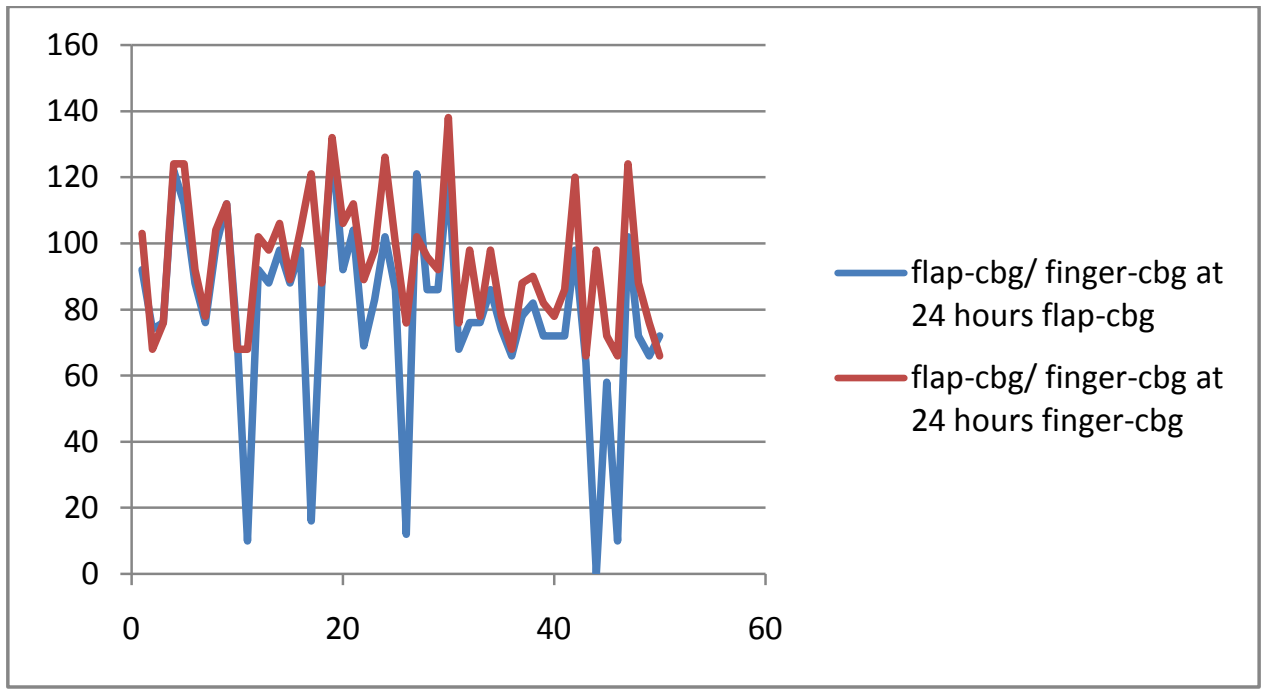

Detection of postoperative complication earlier can prevent flap loss. Despite the results further detailed study is necessary.

\section{Discussion}

Monitoring the BK flap after Bk amputation is of vital importance especially during the first few hours, because the timing of reoperation may determine flap salvage or loss ${ }^{8}$. The use of this objective measurement can reduce the need for human resources.

Flap blood glucose level of $62 \mathrm{mg} / \mathrm{dl}^{9}$, for detection of flap survival had a sensitivity, specificity and positive predictive value of $86.66 \%, 100 \%, 100 \%$ at 0 hour, $100 \%, 100 \%, 100 \%$ at 6 hours and $97.77 \%, 100 \%$, $100 \%$ at 24 hours respectively. More studies with more data series are needed to determine the exact value. The present study confirms a decrease in capillary glucose in all 5 flaps which underwent necrosis and gangrene in the first 24 hours after surgery. Other complications such as infection cannot be detected using capillary blood glucose measurements. Therefore further evaluation of postoperative changes in flap survival is recommended.

\section{Conclusion}

This simple and cheap technique could be used as a routine technique in monitoring BK amputation flaps and predict flap survival in BK amputation patients along with the routine clinical evaluation.

\section{References}

[1]. Wilson AB Jr, eds. Amputation: Surgical Practice and Patient Management. St Louis, Mo: Butterworth-Heinemann Medical; 1996.ebruary 2015. External link in |website= (help).

[2]. "What Is Peripheral Arterial Disease?". http://www.nhlbi.nih.gov/. August 2, 2011. Retrieved 25 FMurdoch G,

[3]. Harry Gouvas: "Accidents and Massive Disasters", editions of Greek Red Cross, 2000

[4]. Wise JB, Talmor M, Hoffman LA, Gayle LB. Postoperative monitoring of micro vascular tissue transplants with an implantable Doppler probe. Plast Reconstr Surg. 2000; 105(6):2279-80.

[5]. Kamolz LP, Giovanoli P, Haslik W, Koller R, Frey M. Continuous free-flap monitoring with tissue-oxygen measurements: threeyear experience. J Reconstr Microsurg. 2002; 18(6):487-91.

[6]. Wilson JL, Morritt AN, Morrison WA. Avoiding complications. In: WeiFC, Mardini S, eds. Flaps and reconstructive surgery. Philadelphia: Saunders; 2009. p.117-24.

[7]. Evans BC, Evans GR. Micro vascular surgery. Plast Reconstr Surg. 2007; 119(2):18e-30e.

[8]. Novakovic D, Patel RS, Goldstein DP, Gullane PJ. Salvage of failed free flaps used in head and neck reconstruction. Head Neck Oncol. 2009; 1:33.

[9]. Hara H, Mihara M, Iida T, Narushima M, Todokoro T, Yamamoto T, et al. Blood glucose measurement for flap monitoring to salvage flaps from venous thrombosis. J Plast Reconstr Aesthet Surg. 2012; 65(5):616-9. 